\title{
Intelligent On-line Monitoring Technology for Capacitance and Dielectric Loss of Electrical Equipment
}

\author{
Junfeng Gui* \\ School of Electrical Engineering, Beijing Jiaotong University, Beijing 100044, PR.China \\ k2011@139.com
}

Keywords: Capacitance; dielectric loss; on-line monitoring; FFT

\begin{abstract}
In order to ensure the safe operation of capacitive equipment in substations, the on-line monitoring technology of capacitance and dielectric loss is implemented. In this paper, the phase comparison method and FFT analysis are used to obtain the insulation parameters such as capacitance and dielectric. Data are uploaded to the station terminal integrated processing unit through the field bus, and the remote diagnosis center is uploaded through the IEC61850 mode for data presentation and analysis diagnosis. The insulation defects of electrical equipment are diagnosed at the scene, and major accidents are avoided in time.
\end{abstract}

\section{Introduction}

Capacitive equipments are important parts of high voltage substations, including power capacitors and bushing, current and voltage transformer and others. It is necessary to ensure their safe operation. Capacitance and dielectric loss are suitable parameters for capacitive equipment's judgment. At present, their off-line testing is widely carried out, but the on-line monitoring technology is rarely implementing because of the difficulty on phase measuring and fault diagnosis.

In this paper, the zero flux current sensor with high precision and high stability is used for measuring. And the high speed FFT digital processing technology is used to solve the problem of dielectric loss measurement of capacitive equipment, which makes the monitoring data have a strong comparability. Then the data acquisition and processing function of the system is realized, and the fault diagnosis of the capacitance device is carried out.

\section{Online Measurement of Dielectric Loss and Capacitance}

In order to realize online monitoring of insulation parameters of capacitive equipment, the key technology is how to acquire and calculate the phase difference of two fundamental frequency current signals accurately.

In the early stage, the "zero crossing" technology based on analog signal processing was used. The time difference between two signals was obtained by the counter method, and then the phase difference was converted according to the period of signal. The method requires higher stability of the hardware and circuit. The internal drift of the circuit and the influence of harmonic interference are all difficult to overcome. In this paper, the digital FFT method is used to obtain the difference of signal. The greatest advantage of this method is that the complex analog processing circuit is not needed, the stability of long-term work can be guaranteed, and the influence of harmonic interference can be effectively suppressed.

The insulation defect monitoring of capacitive equipment is realized by the CT unit, as shown in Figure 1. Each CT unit can monitor 3-phase capacitance equipment as a suit. Many suits can be connected through a field bus when monitoring multiple sets of capacitive equipment. The high precision zero-flux current sensor measures the signal from the ground wire. The fundamental phase of the signal can be carried out by digital FFT processing. 


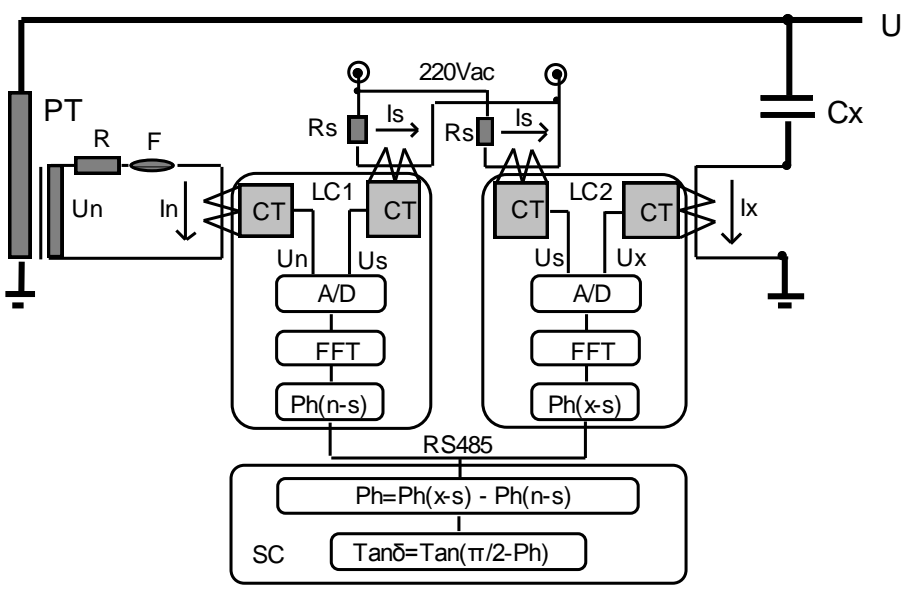

Figure 1. Basic principle of system measurement

The specific measurement principle is shown in Figure 1. shows that the secondary voltage signal Un of the PT is converted to the current signal In through the resistance R. In is detected by the CT in local measurement unit LC1 installed below the PT. And the current signal Ix of the capacitive equipment Cx is detected by the local measurement unit LC2. Under the control of the summit controller(SC), the two signal acquisition units LC1 and LC2 start simultaneously. The analog voltage signal output from the sensor is synchronized with the sampling and FFT conversion processing. Then the fundamental phase $\mathrm{Ph}(\mathrm{N}-\mathrm{S})$ and $\mathrm{Ph}(\mathrm{X}-\mathrm{S})$ of the input signal Un and Ux relative to the 220VAC working power Us are obtained. SC needs only to read the corresponding phase measurement results of LC1 and LC2 through the field communication bus. And it can be calculated that the phase difference $\mathrm{Ph}$ of the terminal current signal Ix of the capacitive equipment relative to the bus voltage Un. Finally, the value of the dielectric loss (dielectric loss angular tangent) and the capacitance $\mathrm{Cx}$, will be got.

\section{Functions of the System}

The terminal integrated processing unit of the system has the following functions:

Data collection: it collects data from each local monitoring unit, and statistical processing or analysis of monitoring data.

Status indication: it has the function of real-time display of operation state and self-test information of each monitoring unit.

Data storage: it will preserve recent monitoring data and their backup.

Fault alarm: if the data of the monitoring equipment is abnormal, and there are faults in the local monitoring unit or the data communication unit,the system sends out alarm information. Alarm messages can be in various forms of sound, light or electricity, and it may be sent by short message service.

Data communication: according to the needs, it uploads monitoring data and results by TCP/IP or IEC61850 protocol.

\section{Communication}

Data communication is always the key and difficult technology of on-line monitoring, which restricts its application. In this paper, the data of the intelligent monitoring unit installed on the site are uploaded to the terminal integrated processing unit through the CAN2.0B field bus. And the terminal integrated processing unit processes the field monitoring data according to the IEC61850 data model. Data will be transfer to the remote diagnostic center by mode of IEC61850 protocol. All kinds of information can be fully displayed by data analysis and diagnosis.

In addition, the software system of on-line monitoring device has functions of remote communication, data management, backup and analysis, diagnosis and early warning, which can be installed and used in the existing platform of the power-grid user. 
The terminal integrated processing unit has 2 field-bus interfaces and 1 remote network interface. The field-bus interface uses CAN2.0B to manage the site bus network in the station. It controls and coordinates the working state of monitoring units in the station and accept monitoring data. The remote interface supports the complete TCP/IP protocol. It can transmit monitoring data to the remote center through the WAN, the LAN or the proprietary network. The IEC61850 communication processor can be modeled in accordance with the definition defined by the user and uploaded to remote diagnostic center.

\section{Functions of the software}

It can monitor all equipments in a substation and send out alarm information from individual faults. When the device data is abnormal, it displays the fault directly. Double-click the name of the substation with the mouse, all monitored equipments in the substation can be expanded and sorted by its type list. Moving the mouse to the icon of the equipment will display the detailed information of the monitored equipment.

State monitoring and analysis software provides the following functions.

Data analysis: the monitoring data which is used to display the characteristic parameters of the equipment, such as waveform and the changing trend of the monitoring data.

Data query: a variety of query methods will be provided and displayed in a tabular way.

Diagnostic report: the state of the monitored equipment and the operation of the monitoring system itself will be displayed with intelligent diagnosis conclusions.

The system also has regular functions including data saving and report printing.

\section{Conclusion}

The on-line monitoring technology of capacitance equipment is studied in this paper.

The digital FFT method is used to solve the difficulties of phase measurement, and on-line measurement of capacitance and dielectric loss is achieved.

Realize data can be remote transferred by field bus, Ethernet and IEC61850 protocol.

There are many functions such as analysis, query and early warning in the system.

In the diagnosis of capacitive equipment, it has accumulated abundant online diagnosis experience. At present, the insulation defects of 14 electrical equipment have been detected and accurately diagnosed, and the occurrence of major accidents is avoided in time.

An example is shown in Figure. 2. The current transformer is monitored in a substation. Data of the A phase at the beginning of the year is as the benchmark. It is found that the dielectric loss of $\mathrm{C}$ phase has been rising steadily since February, 2017.

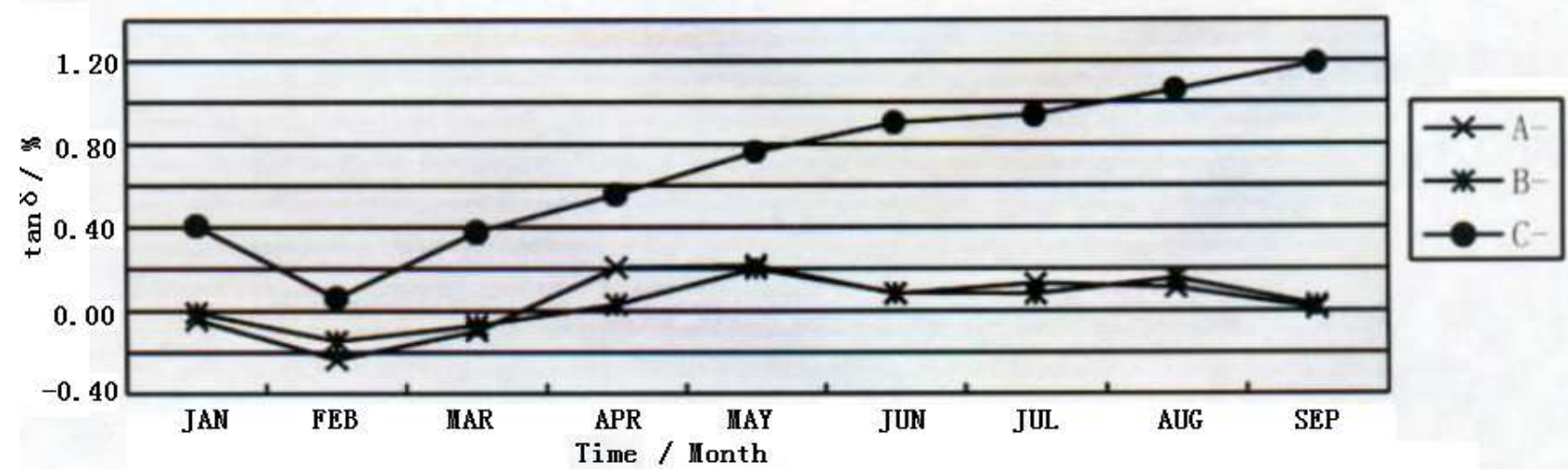

Figure 2. Variation of dielectric loss of three phase current transformers in 2017 


\section{Acknowledgements}

This study is supported by the Fundamental Research Funds for the Central Universities of China (E16JB00100).

\section{References}

[1] S.C. Zi, P. Tian, X Li: Research on on-line monitoring system for dielectric loss of capacitive electrical equipments, Jilin Electric Power, Vol.46(2018)No.2, p35-38.

[2] T. Yan, J. Li: Design of Dielectric Loss of Capacitive Equipment Online Monitoring Device Based on ZigBee, Techniques of Automation and Applications, Vol.34(2015)No.2, p37.

[3] T. Song, A Novel Method for Online Monitoring of Dielectric Loss Factor, Electrical Measurement and Instrumentation, Vol.22(2014)No.1, p119.

[4] P. Wang, M. R. Raghuveer, W. McDermid, J. C. Bromley: A Digital Technique for the On - Line Measurement of Dissipation Factor and Capacitance, IEEE Trans. Dielectrics and Electrical Insulation, Vol.28(2001)No.8, p228.

[5] Z. Wang, X.B Huang, T. Deng: Dielectric Loss of Transformer Bushing On-line Monitoring Apparatus, Guangdong Electric Power, Vol. 13(2013)No.7, p72.

[6] T. Zhao, Q.M. Li, P. Chen: Robust algorithm for accurately monitoring the dielectric loss factor, Journal of Tsinghua University(Science and Technology), Vol.42(2005)No.7, p881.

[7] Z.L. Zhou, Y.Q. Xue, M.W. Chen: On-Line Equipment Monitoring Accuracy Base on Software Method to Improve Dielectric Loss Angle, Electrotechnics Electric, Vol.10(2011)No.2, p39. 